\title{
Profilaxis de la tromboembolia venosa en pacientes colombianos de tratamiento médico o quirúrgico: resultados para Colombia del estudio ENDORSE
}

\author{
Rodolfo J. Dennis ${ }^{1,2}$, Jairo H. Roa ${ }^{3}$, Juan Villadiego ${ }^{4}$, Freddy Méndez ${ }^{5}$, Elías Vieda ${ }^{6}$, Héctor Restrepo \\ 1 Departamento Medicina Interna, Fundación Cardioinfantil-Instituto de Cardiología, Bogotá, D.C., Colombia \\ 2 Departamento de Epidemiología Clínica y Bioestadística, Pontificia Universidad Javeriana, Bogotá, D.C., \\ Colombia \\ 3 Departamento de Medicina Interna, Fundación Santafé de Bogotá, Bogotá, D.C., Colombia \\ 4 Departamento de Medicina Interna, Clínica Rafael Uribe Uribe, Cali, Colombia \\ 5 Departamento de Medicina Interna, Clínica Soma, Medellín, Colombia \\ 6 Unidad de Terapia Intensiva, Departamento de Medicina Interna, Hospital Universitario del Valle, Cali, Colombia \\ 7 Departamento de Investigaciones, Fundación Cardioinfantil-Instituto de Cardiología, Bogotá, D.C., Colombia
}

Introducción. La profilaxis en pacientes de tratamiento médico o quirúrgico con riesgo de tromboembolia venosa, requiere ser evaluada para obtener suficiente información que contribuya a mejorar estas prácticas, para que sean efectivas y eficaces, y ayudar con las medidas necesarias de prevención hospitalaria de la tromboembolia venosa.

Objetivo. Evaluar el riesgo de tromboembolia venosa y de los hábitos de profilaxis en pacientes de tratamiento médico o quirúrgico hospitalizados en Colombia y compararlos con los de los centros internacionales participantes en el estudio ENDORSE.

Materiales y métodos. Las instituciones fueron escogidas al azar. Se evaluaron todas las historias clínicas de pacientes de tratamiento médico o quirúrgico. Se utilizaron las guías del 2004 del American College of Chest Physicians para evaluar el riesgo de tromboembolia venosa y lo apropiado de la profilaxis.

Resultados. Se evaluaron 761 pacientes: 218 de tratamiento quirúrgico y 543 de tratamiento médico, de cinco centros hospitalarios de Colombia. De esta población, 49 \% se encontraba en riesgo de tromboembolia venosa ( $40 \%$ de los de tratamiento médico y $72 \%$ de los de tratamiento quirúrgico), en comparación con $52 \%$ de la muestra internacional. La profilaxis fue significativamente mayor en los pacientes de tratamiento médico en riesgo $(63,7 \%, \mathrm{n}=137)$, que en los de tratamiento quirúrgico $(48,4$ $\%, n=76, P=0,01)$. En comparación con la muestra internacional, la profilaxis en los de tratamiento médico fue significativamente superior $(63,7 \% V s .39,5 \%, P=0,003)$, a diferencia de los de tratamiento quirúrgico $(48,4 \%$ Vs. 58,5\%, $\mathrm{P}=0,02)$.

Conclusiones. Los resultados muestran que en los centros hospitalarios de Colombia se trataron pacientes con riesgo de tromboembolia venosa en forma similar al resto de los países participantes en el estudio ENDORSE, y que se hizo más profilaxis apropiada en pacientes de tratamiento médico. Sin embargo, se requiere mejorar la utilización de profilaxis en pacientes hospitalizados.

Palabras clave: tromboembolia venosa/epidemiologia, trombosis/prevención y control, control de riesgo, cirugía general, prescripciones de medicamentos.

Venous thromboembolism prophylaxis in Colombian surgical and medical patients: Results for Colombia of the ENDORSE study

Introduction. More information is needed on the risk of venous thromboembolism in the hospital setting, and on patterns of use of thromboprophylaxis, as advocated in consensus guidelines. ENDORSE was an international study aimed at evaluating hospital venous thromboembolism prevention practices in medical and surgical patientes.

Objectives. The risk of venous thromboembolism was evaluated along with the use of thromboprophylaxis in hospitalized medical and surgical subjects; these data were compared with the international sample from the ENDORSE study.

Materials and methods. Participating institutions in Colombia were selected arbitrarily. The medical charts for medical and surgical patients were evaluated randomly. The 2004 American College of Chest Physician guidelines were used to evaluate risk of venous thromboembolism and adherence to recommended thromboprophylaxis regimens.

Results. The study included 761 subjects (218 surgical, 543 medical) located in five acute care hospitals; $49 \%$ of these subjects were considered at risk of venous thromboembolism $(40 \%$ medical, 
$72 \%$ surgical), compared with $52 \%$ in the international sample. Prophylaxis use was higher in medical patients at risk $(63.7 \%, \mathrm{n}=137)$ than in surgical patients $(48.4 \%, \mathrm{n}=76 ; p=0.01)$. Compared with the international sample, the use of prophylaxis in Colombia was greater in medical patients $(63.7 \%$ vs. $39.5 \%, p=0.003)$, but lower in surgical patients $(48.4 \%$ vs. $58.5 \%, p=0.02)$.

Conclusions. Participating Colombian centers treat patients at risk of venous thromboembolism similarly to other participant countries, but appropriate prophylaxis was prescribed more frequently to medical patients. Greater efforts are needed, both in Colombia and around the world, to improve rates of appropriate venous thromboembolism prophylaxis in at-risk subjects.

Key words: venous thromboembolism/epidemiology, thrombosis/prevention \& control, risk management, general surgery, drug prescriptions.

La tromboembolia venosa puede complicar el curso de los pacientes hospitalizados por indicación médica o quirúrgica, aumentando tiempos de estancia y llevando a complicaciones graves, inclusive aumentando la mortalidad, como se ha documentado en Colombia y en otros países $(1,2)$. Para ayudar con las medidas necesarias de prevención hospitalaria de la tromboembolia venosa, existen guías de práctica clínica nacionales (3) e internacionales $(4,5)$ para la identificación del riesgo y las medidas, basadas en la evidencia, que se consideran seguras y eficaces, dependiendo del grado de riesgo de los pacientes.

Varios estudios en diferentes países, incluido Colombia, han demostrado que la aplicación de estas guías y de otras intervenciones están subutilizadas en pacientes que se beneficiarían de ellas (6-8).

No hay muchos estudios a nivel mundial, ni latinoamericano, que hayan evaluado de forma sistemática el manejo apropiado de la profilaxis de la tromboembolia venosa en el paciente hospitalizado por causa médica o quirúrgica, agudamente enfermo. El recientemente publicado estudio IMPROVE (International Medical Prevention Registry on Venous Thromboembolism) $(6,8)$, se diseñó en parte para tratar de evaluar este objetivo. Sin embargo, el estudio IMPROVE tuvo algunas limitaciones: no se evaluaron pacientes del área quirúrgica; la escogencia de centros hospitalarios fue por conveniencia, por lo que las frecuencias observadas de profilaxis pueden no ser generalizables, y los pacientes que se enrolaron en el estudio no fueron el resultado del censo total de la población hospitalaria ni de una muestra al azar, dado que se escogían sólo

\footnotetext{
Correspondencia:

Rodolfo J. Dennis, Departamento de Investigaciones, Fundación Cardioinfantil, Carrera 13b № 163-85, torre $\mathrm{H}$, tercer piso, Bogotá, D.C., Colombia

Teléfono: (571) 667 2727, extensión 6300; fax: (571) 6791236 rdennis@cardioinfantil.org

Recibido: 29/06/10; aceptado:21/02/11
}

los 10 primeros pacientes elegibles en cada mes (cabe la posibilidad que estos pacientes fueran sistemáticamente diferentes de los pacientes que se hospitalizaran el resto del mes).

Estas limitaciones se consideraron en un nuevo estudiomundial, elestudioENDORSE(Epidemiologic International Day for the Evaluation of Patients at Risk of Venous Thrombosis in the Acute Hospital Care Setting), en el que también participaron cinco centros colombianos y cuyos resultados globales se publicaron recientemente (9).

El presente estudio muestra los resultados provenientes de los centros colombianos participantes en ENDORSE y compara la información con la de todos los centros participantes en la muestra internacional.

\section{Materiales y métodos}

Este fue un estudio observacional, analítico, de corte transversal, enfocado hacia la evaluación sistemática de la práctica clínica rutinaria de la profilaxis de la tromboembolia venosa en el ámbito hospitalario. El manejo de los pacientes era el establecido por los médicos tratantes. El estudio fue diseñado y coordinado bajo la guía de un comité científico asesor internacional, por el Center for Outcomes Research del School of Medicine, University of Massachusetts, en Worcester, Estados Unidos.

\section{Instituciones elegibles}

Los centros hospitalarios participantes en el estudio, incluyendo los colombianos, fueron escogidos al azar de listas nacionales de instituciones prestadoras de servicios de salud que tuvieran más de 50 camas, programaran procedimientos de cirugía mayor y hospitalizaran pacientes de tratamiento médico con enfermedades agudas o crónicas exacerbadas. Se excluyeron hospitales con pacientes crónicos o de una sola especialidad.

La lista de hospitales elegibles en cada país era enviada al Center for Outcomes Research donde 
se seleccionaba la muestra en forma aleatoria generada por un programa estadístico (SAS®, versión 9.1). Una vez se conocía el resultado, la lista era enviada al investigador principal en cada país, quien contactaba a cada uno de los centros seleccionados.

\section{Camas y salas de estudio}

En cada hospital, para ser elegible, una cama - sala debía estar ocupada por pacientes de tratamiento quirúrgico (ortopedia, cirugía general) o de tratamiento médico. Se excluyeron sistemáticamente camas o salas dedicadas al cuidado de pacientes psiquiátricos, pediátricos, obstétricos, en rehabilitación, en cuidados crónicos o paliativos, o en urgencias.

\section{Sujetos de estudio}

Los pacientes de tratamiento quirúrgico elegibles debían tener más de 18 años y los de tratamiento médico debían tener mínimo 40 años para participar en el estudio. Se excluyeron del estudio los pacientes que ingresaron para tratamiento de tromboembolia venosa o que estuvieran recibiendo desde el ingreso dosis terapéuticas de anticoagulantes 0 trombolíticos, o que estuvieran participando en un estudio experimental terapéutico.

Las historias clínicas de los sujetos elegibles eran evaluadas para identificar la presencia de factores de riesgo de tromboembolia venosa de acuerdo con las guías del American College of Chest Physicians (ACCP) del 2004, las cuales se basan en evidencia (10-12) y son categorizadas por grados de recomendación (13). Los pacientes quirúrgicos se clasificaban en riesgo bajo, moderado, alto y muy alto, teniendo en cuenta la edad, tipo y duración de la cirugía y tipo/duración de la anestesia. Los tipos de riesgo de profilaxis se definieron de acuerdo con las recomendaciones específicas de la misma guía (13).

Los pacientes que tuvieran contraindicación para anticoagulación profiláctica, así existiera la indicación, si presentaban o desarrollaban durante la hospitalización sangrado intracraneal, daño hepático, sangrado al ingreso a la hospitalización, úlcera gastroduodenal activa o discrasia hematológica conocida, no fueron incluidos en este estudio.

\section{Recolección y manejo de datos}

Los coordinadores (médicos, enfermeras, otro personal del área de la salud) eran entrenados previamente en la recolección de información, con base en un manual explícitamente diseñado para este propósito. El personal del Center for Outcomes Research viajó a cada país para una sesión de un día de entrenamiento de coordinadores e investigadores en cada hospital.

La calidad de los datos se documentó y supervisó durante el estudio. El coordinador del estudio en cada centro mantenía una bitácora de los pacientes elegibles incluidos, excluidos y las razones para su exclusión. Una copia era enviada al Center for Outcomes Research. Los formatos con páginas faltantes, ilegibles, datos faltantes, datos inválidos o no concordantes, se investigaban y resolvían.

Se solicitó a los investigadores que recolectaran la información de los pacientes de cada sala o ala hospitalaria en un tiempo máximo de 14 días; estos datos fueron recolectados en formatos estandarizados de reportes de caso y enviados al Center for Outcomes Research para su inclusión en la base de datos. La información incluía: datos demográficos básicos, antecedentes médicos previos, diagnósticos al ingreso y posteriores, factores de riesgo para tromboembolia venosa y para sangrado (predefinidos), duración de la estancia, medicamentos, historia y profilaxis intrahospitalaria formulada.

\section{Análisis estadístico}

El análisis se hizo por medio del paquete estadístico SAS®, versión 9.1. Para evaluar el riesgo de tromboembolia venosa, asumiendo una prevalencia estimada de $25 \%$ con un margen de error de $4 \%$ y error alfa de $95 \%$, era necesario un mínimo de 450 sujetos por país. Se hizo un análisis descriptivo de la población total, estratificada por país y centro hospitalario, que incluía las medidas usuales de tendencia central (medianas, promedios) y de dispersión de los datos (rango y rango intercuartil), así como porcentajes e intervalos de confianza de $95 \%$.

Lo apropiado de la profilaxis según el riesgo de tromboembolia venosa, con base en las recomendaciones del consenso del American College of Chest Physicians, se comparó con lo observado para el perfil de riesgo de los pacientes incluidos en este estudio. Los análisis estadísticos realizados evaluaron la desviación de la hipótesis nula de no diferencias en tasas de profilaxis apropiada. Las distribuciones de la $\chi^{2}$ y el test exacto de Fisher, se utilizaron debido a la naturaleza dicotómica de la mayoría de las variables y a los desenlaces dicotómicos que se 
evaluaron (profilaxis sí/no). Se rechazó la hipótesis nula de no diferencias entre grupos a un nivel de significación de $5 \%$. No se hicieron consideraciones por múltiples pruebas de hipótesis.

\section{Consideraciones éticas}

Dado que no hubo contacto directo con pacientes, recolección de información sensible, ni datos de identificación directa del paciente o del centro, el protocolo general del estudio fue aprobado por los respectivos comités de ética en investigaciones de cada centro hospitalario de Colombia, sin necesidad de consentimiento informado.

\section{Resultados}

El reclutamiento de pacientes para ENDORSE empezó en agosto de 2006 y terminó en enero de 2007, en 32 países y 358 instituciones hospitalarias de seis continentes, de las cuales, el $44 \%$ eran académicas y la mediana de camas de 352 , con un rango entre 169 y 1120 (9). El número de pacientes incluidos en el mundo fue de 68.183. En los cinco centros colombianos: Clínica Rafael Uribe Uribe (Cali), Clínica Soma (Medellín), Fundación Cardioinfantil (Bogotá, D.C.), Fundación Santafé de Bogotá (Bogotá, D.C.) y Hospital Universitario del Valle (Cali). Se evaluaron 884 pacientes en camas o salas elegibles, de los cuales, se incluyeron 761 pacientes, correspondientes a $86 \%$ (figura 1 ).

Del total de la muestra colombiana, 48,9 \% ( $n=372$, $\left.\mathrm{IC}_{95 \%}: 45-52 \%\right)$ se encontraron con riesgo de tromboembolia venosa, en comparación con $51,8 \%$ en la muestra internacional $(P=0,20)$. Sin embargo, existieron diferencias en el riesgo entre pacientes de tratamiento quirúrgico o médico en la muestra colombiana, y entre ésta y la internacional.

En Colombia, $72 \%$ de los pacientes de tratamiento quirúrgico cumplieron criterios de riesgo, contra $39,6 \%$ en los de tratamiento médico $(P=0,003)$. En la muestra internacional, el riesgo en los pacientes de tratamiento quirúrgico fue menor que en los colombianos ( $64 \%, P=0,02)$, y algo mayor en los de tratamiento médico $(41,5 \%)$, pero sin significancia estadística con respecto a Colombia $(\mathrm{P}=0,34)$.

En el cuadro 1 se presentan las diferencias entre los pacientes colombianos de tratamiento médico o quirúrgico en cuanto a características generales, condiciones presentes antes de la hospitalización y durante ella. En general, se observa un mayor número de pacientes de tratamiento médico frente a los de tratamiento quirúrgico; los primeros presentaron con mayor frecuencia enfermedad

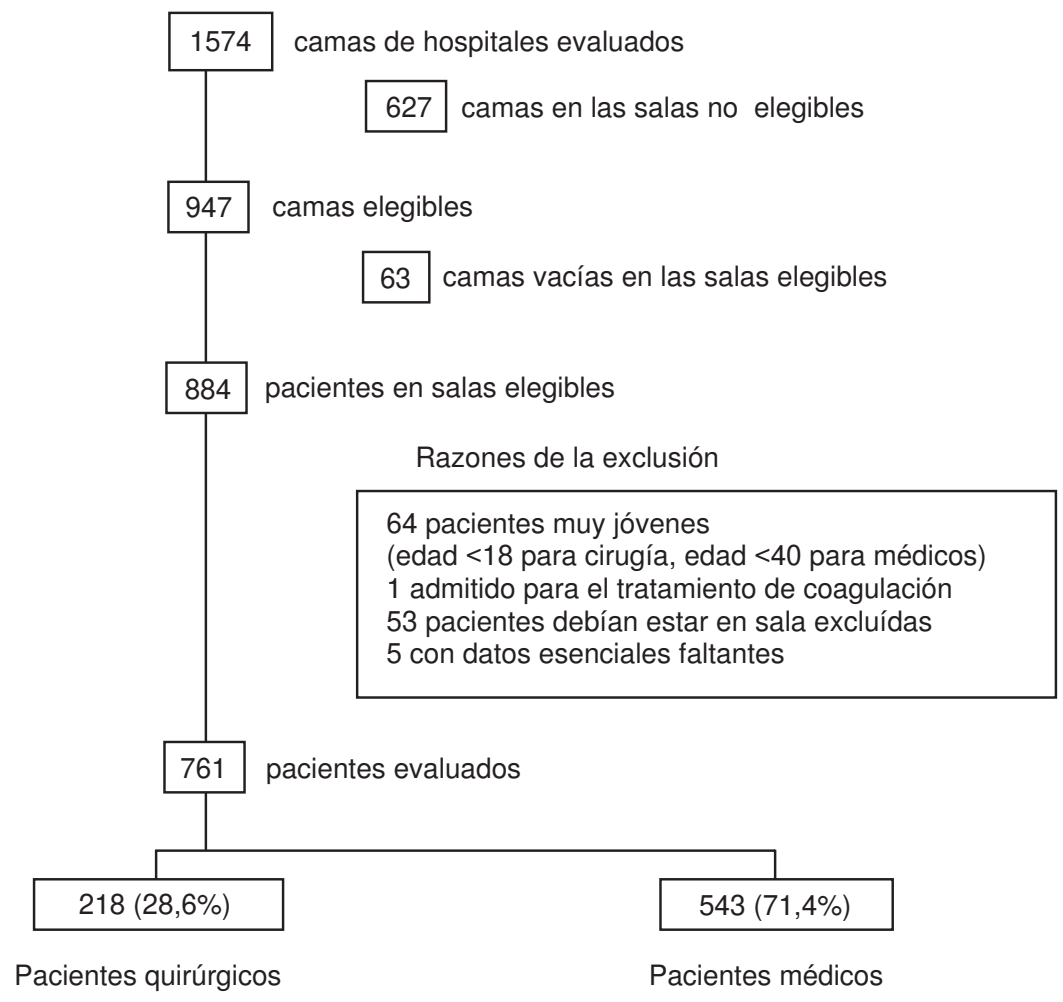

Figura 1. Selección de la población en estudio y razones para exclusión. 
Cuadro 1. Características generales de los pacientes de tratamiento médico o quirúrgico en riesgo de tromboembolia venosa.

\begin{tabular}{|c|c|c|c|}
\hline Características de los pacientes & $\begin{array}{c}\text { Quirúrgicos } \\
n=157\end{array}$ & $\begin{array}{c}\text { Médicos } \\
n=215\end{array}$ & $\begin{array}{c}\text { Total } \\
\mathrm{n}=372\end{array}$ \\
\hline Mujeres & $82(52,6)$ & $111(51,6)$ & $193 \quad(52)$ \\
\hline Edad (mediana, años) & $157 \quad(63)$ & $215 \quad(69)$ & 372 (66) \\
\hline Peso (mediana, kg) & $77 \quad(65)$ & $68(62,5)$ & $145 \quad(65)$ \\
\hline Estatura (mediana, cm) & $55 \quad(160)$ & $62(160)$ & 117 (160) \\
\hline Índice de masa corporal (mediana, $\mathrm{kg} / \mathrm{m}^{2}$ ) & $44(24,1)$ & $52(24,6)$ & $96(24,5)$ \\
\hline Duración de la hospitalización (días) & $155 \quad$ (3) & $213 \quad(4)$ & $368(4,0)$ \\
\hline Condiciones de los pacientes antes de admisión & n $\quad(\%)$ & $(\%)$ & n $\quad(\%)$ \\
\hline Tromboembolia previa & $5 \quad(3,3)$ & $3 \quad(1,4)$ & $8(2,2)$ \\
\hline Trombofilia & $1 \quad(0,7)$ & (0) & $1 \quad(0,3)$ \\
\hline Insuficiencia venosa & $5 \quad(3,3)$ & $15 \quad(7,0)$ & $20 \quad(5,5)$ \\
\hline Enfermedad pulmonar crónica & $8 \quad(5,3)$ & $59(27,7)$ & $67(18,4)$ \\
\hline Inmovilidad prolongada & $4 \quad(2,6)$ & $20 \quad(9,4)$ & $24(6,6)$ \\
\hline Obesidad & $7 \quad(4,6)$ & $20 \quad(9,4)$ & $27 \quad(7,4)$ \\
\hline Uso de anticonceptivos & $1 \quad(0,7)$ & $(0)$ & $1 \quad(0,3)$ \\
\hline Falla cardiaca crónica & $9 \quad(6,0)$ & $33(15,5)$ & $42(11,5)$ \\
\hline Condiciones presentes durante la hospitalización & n $\quad(\%)$ & $(\%)$ & n $\quad(\%)$ \\
\hline Falla cardiaca aguda (NYHA clase III o IV) & $2 \quad(1,3)$ & $20 \quad(9,3)$ & $22(5,9)$ \\
\hline Choque isquémico & $1 \quad(0,6)$ & $11 \quad(5,1)$ & $12(3,2)$ \\
\hline Choque hemorrágico & $1 \quad(0,6)$ & $1 \quad(0,5)$ & $2(0,5)$ \\
\hline Otras enfermedades cardiovasculares & $9 \quad(5,7)$ & $27(12,6)$ & $36(89,7)$ \\
\hline Enfermedades hematológicas & (0) & $3 \quad(1,4)$ & $3(0,8)$ \\
\hline Enfermedad respiratoria no infecciosa & $2 \quad(1,3)$ & $64(29,8)$ & $66(17,7)$ \\
\hline Infección pulmonar & $3 \quad(1,9)$ & $51 \quad(23,7)$ & $54(14,5)$ \\
\hline Neoplasia maligna activa & $9 \quad(5,7)$ & $13(6,0)$ & $22(5,9)$ \\
\hline Infección no respiratoria & $18(11,5)$ & $54(25,1)$ & $72(19,0)$ \\
\hline Enfermedad reumatológica o infecciosa & $3 \quad(1,9)$ & $2(0,9)$ & $5(1,3)$ \\
\hline Enfermedad neurológica & $9 \quad(5,7)$ & $15 \quad(7,0)$ & $24(6,5)$ \\
\hline Enfermedad renal & $12(7,6)$ & $13 \quad(6,0)$ & $25(6,7)$ \\
\hline Enfermedad endocrina o metabólica & $5 \quad(3,2)$ & $12 \quad(5,6)$ & $17 \quad(8,1)$ \\
\hline Enfermedad gastrointestina o hepatobiliar & $21 \quad(13,4)$ & $9 \quad(4,2)$ & $30 \quad(8,1)$ \\
\hline Otras condiciones médicas & $47 \quad 29,9)$ & $23(11,0)$ & $7018,8)$ \\
\hline
\end{tabular}

pulmonar crónica, falla cardíaca, enfermedad cardiovascular e inmovilidad.

En el cuadro 2 se muestran los factores de riesgo para tromboembolia venosa durante la hospitalización y para los sangrados más frecuentes. Fue común la inmovilización completa en los pacientes de tratamiento quirúrgico, así como la utilización de catéteres, la necesidad de asistencia respiratoria mecánica y la disfunción renal en los de tratamiento médico.

En el cuadro 3 se presentan los tipos de cirugía practicada en la muestra quirúrgica, en los que predominaron las intervenciones de cirugía general, ortopedia y trauma.

\section{Frecuencia y tipos de profilaxis practicada}

De los pacientes con riesgo de tromboembolia venosa, 54,8 \% ( $\left.\mathrm{n}=204, \mathrm{IC}_{95 \%}: 51-58 \%\right)$ recibieron profilaxis en Colombia, en comparación con $50,2 \%$ en la muestra internacional $(P=0,1)$.
Se encontraron diferencias en frecuencia de profilaxis en pacientes en la muestra colombiana, y entre ésta y la internacional; cuando se clasificó por tipo de paciente (de tratamiento quirúrgico Vs. de tratamiento médico), la profilaxis fue significativamente mayor en los de tratamiento médico en riesgo $(63,7 \%, \mathrm{n}: 137)$, que en los de tratamiento quirúrgico $(48,4 \%, \mathrm{n}=76, \mathrm{P}=0,01)$.

En comparación con la muestra internacional, la profilaxis apropiada en pacientes de tratamiento médico en riesgo en Colombia fue significativamente superior (64 \% Vs. $40 \%, \mathrm{P}=0,003)$, y la segunda más alta entre todos los países participantes (9). La frecuencia de profilaxis apropiada en pacientes de tratamiento quirúrgico colombianos en riesgo fue significativamente inferior a la observada en la muestra mundial, (43\% Vs. $59 \%, \mathrm{P}=0,02)$, en el puesto 23/32(9).

En el cuadro 4 se muestra la frecuencia de profilaxis por tipo de paciente de tratamiento 
Cuadro 2. Factores de riesgo para sangrado al ingreso y para tromboembolia venosa durante la hospitalización.

\begin{tabular}{|c|c|c|}
\hline Factores de riesgo para sangrado al ingreso & $\begin{array}{l}\text { Quirúrgicos } \\
\text { n }(\%)\end{array}$ & $\begin{array}{l}\text { Médicos } \\
\mathrm{n} \quad(\%)\end{array}$ \\
\hline Falla renal significativa & $8 \quad(5,1)$ & $24 \quad(11,2)$ \\
\hline Hemorragia intracraneal & $4 \quad(2,5)$ & $\begin{array}{ll}0 & (0)\end{array}$ \\
\hline Bajo recuento de plaquetas & $0 \quad(0)$ & $1 \quad(0,5)$ \\
\hline Falla hepática & $2 \quad(1,3)$ & (0) \\
\hline Sangrado & $8 \quad(5,1)$ & $9 \quad(4,2)$ \\
\hline úlcera gastrointestinal activa & $6 \quad(3,8)$ & $4 \quad(1,9)$ \\
\hline Uso de ácido acetilsalicílico & $8 \quad(5,1)$ & $43(20,0)$ \\
\hline Otros antiinflamatorios & $6 \quad(3,8)$ & $14 \quad(6,5)$ \\
\hline $\begin{array}{l}\text { Factores de riesgo adicionales para tromboembolia venosa } \\
\text { durante la hospitalización }\end{array}$ & n $\quad(\%)$ & n $\quad(\%)$ \\
\hline Admitidos a cuidados intensivos & $34(21,7)$ & $45 \quad(20,9)$ \\
\hline Catéter venoso central & $21 \quad(13,4)$ & $17(7,9)$ \\
\hline Asistencia respiratoria mecánica & $16(10,2)$ & $10 \quad(4,7)$ \\
\hline Inmovilización con privilegios de baño & $20(12,7)$ & $82(38,1)$ \\
\hline Inmovilización completa & $65(41,4)$ & $64 \quad(29,8)$ \\
\hline Tratamiento de cáncer & $4 \quad(2,5)$ & $3 \quad(1,4)$ \\
\hline
\end{tabular}

Cuadro 3. Tipo de cirugía practicada en la muestra.

\begin{tabular}{lcc}
\hline & Frecuencia & Porcentaje \\
\hline Reemplazo de cadera & 5 & 3,2 \\
Reemplazo de rodilla & 3 & 1,9 \\
Reducción de fractura de cadera & 4 & 2,5 \\
Artroscopia curativa & 1 & 0,6 \\
Otros traumas ortopédicos & 16 & 10,2 \\
Colon o intestino delgado & 13 & 8,3 \\
Recto-sigmoidea & 1 & 0,6 \\
Gástrica & 16 & 10,2 \\
Hepatobiliar & 7 & 4,5 \\
Urológica & 22 & 14,0 \\
Vascular & 10 & 6,4 \\
Torácica & 4 & 2,5 \\
Ginecológica & 12 & 7,6 \\
Otra cirugía mayor & 25 & 15,9 \\
Admitido por trauma mayor & 18 & 11,5 \\
sin cirugía & & \\
\hline
\end{tabular}

quirúrgico y de tratamiento médico. La muestra fue pequeña y no permitió concluir por condiciones o especialidades.

En el cuadro 5 se consignan los diferentes tipos de profilaxis (farmacológica y mecánica) más frecuentes, estratificadas por tipo de paciente (de tratamiento quirúrgico Vs. de tratamiento médico), contra la muestra internacional. En general, fue más común la utilización de medias de compresión graduada en los colombianos de tratamiento quirúrgico que en los de tratamiento médico; en éstos predominó la profilaxis farmacológica.

\section{Discusión}

ENDORSE es uno de los pocos estudios existentes con la mayor muestra de pacientes hospitalizados de tratamiento médico o quirúrgico, que brinda información sobre el riesgo de tromboembolia venosa, la frecuencia, el tipo y la pertinencia de la profilaxis en éstos y lo compara con datos de centros similares. Deben discutirse las limitaciones de los métodos y, por ende, las conclusiones del estudio ENDORSE. Debido a que la recopilación de la información se tomó directamente de las historias clínicas y no de entrevistas con el paciente, se podría generar una mala clasificación de información relevante para categorizar el riesgo de tromboembolia venosa y la decisión de profilaxis, como factores de riesgo de trombosis y de sangrado no consignados en la historia. Sin embargo, puede argumentarse que la misma información fue la que tuvo disponible el clínico para tomar la decisión. Además, el diseño de corte transversal sólo brinda información sobre el perfil de riesgo del paciente y la pertinencia de la profilaxis hasta el momento de la evaluación; no se pueden hacer inferencias sobre el perfil de riesgo y lo apropiado de la profilaxis posteriormente en la hospitalización del sujeto. Finalmente, es posible que el personal de salud, al sentirse evaluado, haya cambiado sus hábitos de profilaxis hacia patrones más apropiados durante la duración de la recolección de la información. Sin embargo, esto es poco probable debido a la naturaleza transversal del diseño y al corto tiempo de recolección de la información.

ENDORSE muestra que los pacientes en Colombia tienen en promedio un nivel de riesgo de tromboembolia venosa similar al de otras poblaciones de pacientes internacionales hospitalizados; esta frecuencia es alrededor de 
Cuadro 4. Tipo de profilaxis más frecuente, estratificada por tipo de paciente.

\begin{tabular}{|c|c|c|c|c|}
\hline \multirow[t]{2}{*}{ Paciente de tratamiento quirúrgico } & \multicolumn{2}{|c|}{$\begin{array}{l}\text { Recibieron alguna } \\
\text { profilaxis }\end{array}$} & \multicolumn{2}{|c|}{$\begin{array}{l}\text { Profilaxis apropiada } \\
\text { (según criterios } A C C P \text { ) }\end{array}$} \\
\hline & n & $(\%)$ & $\mathbf{n}$ & (\%) \\
\hline Reemplazo de cadera & 4 & $(80)$ & 3 & $(60)$ \\
\hline Reemplazo de rodilla & 2 & $(66,7)$ & 2 & $(66,7)$ \\
\hline Reducción de fractura de cadera & 1 & (25) & 1 & (25) \\
\hline Artroscopia curativa & 1 & $(100)$ & 0 & (0) \\
\hline Otro trauma ortopédico & 5 & $(31,1)$ & 5 & $(31,1)$ \\
\hline Colon/intestino delgado & 9 & $(69,2)$ & 8 & $(61,5)$ \\
\hline Gástrica & 6 & $(37,5)$ & 6 & $(37,5)$ \\
\hline Hepatobiliar & 4 & $(57,1)$ & 3 & $(42,9)$ \\
\hline Urológica & 6 & $(27,3)$ & 2 & $(9,1)$ \\
\hline Vascular & 9 & $(90,0)$ & 9 & $(90,0)$ \\
\hline Torácica & 1 & $(25,0)$ & 0 & (0) \\
\hline Ginecológica & 8 & $(66,7)$ & 8 & $(66,7)$ \\
\hline Otras cirugías & 11 & $(44,0)$ & 11 & $(44,0)$ \\
\hline Trauma mayor sin cirugía & 9 & $(50,0)$ & 9 & $(50,0)$ \\
\hline Paciente de tratamiento médico & $\mathbf{n}$ & $(\%)$ & $\mathbf{n}$ & $(\%)$ \\
\hline Falla renal aguda & 17 & $(85,0)$ & 17 & $(85,0)$ \\
\hline ECV isquémica & 8 & $(72,7)$ & 8 & $(72,7)$ \\
\hline Otra condición cardiovascular & 13 & $(48,1)$ & 13 & $(48,1)$ \\
\hline Enfermedad hematológica & 3 & $(100)$ & 3 & $(100)$ \\
\hline Neoplasia maligna activa & 6 & $(46,2)$ & 6 & $(46,2)$ \\
\hline Enfermedad respiratoria no infecciosa & 48 & $(75,0)$ & 48 & $(75,0)$ \\
\hline Infección pulmonar & 30 & $(58,8)$ & 30 & $(58,8)$ \\
\hline Infección no respiratoria & 34 & $(63,0)$ & 34 & $(63,0)$ \\
\hline Enfermedad reumatológica o inflamatoria & 2 & $(100)$ & 2 & $(100)$ \\
\hline Enfermedad neurológica & 10 & $(66,7)$ & 10 & $(66,7)$ \\
\hline Enfermedad renal & 8 & $(61,5)$ & 8 & $(61,5)$ \\
\hline Enfermedad endocrina o metabólica & 9 & $(7,5)$ & 9 & $(7,5)$ \\
\hline Enfermedad gastrointestinal o hepatobiliar & 5 & $(55,6)$ & 5 & $(55,6)$ \\
\hline Otras condiciones médicas & 13 & $(56,6)$ & 13 & $(56,6)$ \\
\hline
\end{tabular}

ACCP: American College of Chest Physicians; ECV: enfermedad cerebro-vascular

Cuadro 5. Tipo y frecuencia de profilaxis por tipo de paciente (de tratamiento quirúrgico o médico).

\begin{tabular}{lrrrr}
\hline & \multicolumn{2}{c}{ Quirúrgico } & \multicolumn{2}{c}{ Médico } \\
& $\mathbf{n}$ & $(\%)$ & \multicolumn{1}{c}{$\mathbf{n}$} & $(\%)$ \\
\hline Heparina de bajo peso molecular & 56 & $(35,7)$ & 117 & $(54,4)$ \\
Heparina no fraccionada & 9 & $(5,7)$ & 30 & $(14)$ \\
Antagonista de la vitamina K & 1 & $(0,6)$ & 2 & $(0,9)$ \\
Fondaparinux & 2 & $(1,3)$ & 0 & $(0)$ \\
Otros anticoagulantes & 0 & $(0)$ & 0 & $(0)$ \\
Medias de compresión gradual & 24 & $(15,3)$ & 3 & $(1,4)$ \\
Compresión neumática intermitente & 0 & $(0)$ & 0 & $(0)$ \\
\hline
\end{tabular}

$50 \%$ y los pacientes de tratamiento quirúrgico se encuentran en promedio en mayor riesgo que los del área médica. Sin embargo, llama la atención que los pacientes colombianos de tratamiento quirúrgicos incluidos tuvieron un riesgo mayor, no sólo que el de los pacientes de tratamiento médico, sino también, que el de los de tratamiento quirúrgico en la muestra internacional.

De igual forma, ENDORSE demuestra que la profilaxis apropiada en pacientes en riesgo que se beneficiarían de ella, sólo se llevó a cabo en, aproximadamente, la mitad de los evaluados (9).

Un hallazgo importante es que en Colombia la profilaxis apropiada de tromboembolia venosa en pacientes en riesgo fue significativamente más frecuente en los de tratamiento médico; mientras que la profilaxis en los de tratamiento quirúrgico fue menor, inclusive cuando se comparó con lo observado en la muestra internacional para pacientes de tratamiento quirúrgico.

Las tasas de profilaxis en pacientes hospitalarios de tratamiento médico en riesgo de tromboembolia venosa vistas en este estudio (64\%), son comparables a las encontradas para Colombia en el estudio IMPROVE (76\%) en 1.948 pacientes del área médica hace tres años (6) y han aumentado, significativamente, desde un estudio previo practicado hace 15 años (7). Es satisfactorio que ENDORSE confirme que la frecuencia de profilaxis en pacientes del área médica en Colombia no sólo sea significativamente mayor que la de pacientes 
de tratamiento médico en estudios internacionales, realizados con métodos similares como IMPROVE $(6,8)$ y otros (14), sino que es la más alta encontrada entre los 32 países participantes, después de Alemania (9). Sin embargo, la tasa de profilaxis en los pacientes colombianos de tratamiento quirúrgico fue de las más bajas encontradas en el estudio ENDORSE (9).

Sería de utilidad conocer por qué en Colombia se documenta lo contrario a lo publicado en la literatura científica internacional, la cual muestra sistemáticamente que la profilaxis en pacientes quirúrgicos ha sido más alta, mayor número de estudios de alta calidad se han llevado a cabo, y la evidencia a favor de la profilaxis ha sido más contundente y aceptada como el estándar desde hace más de una década.

Es posible, por ejemplo, que la difusión de la información y el tiempo dedicado a desarrollar, implementar y seguir las guías de práctica clínica, hayan sido insuficientes para garantizar un cambio importante en los hábitos de prevención de la tromboembolia venosa en el área quirúrgica en Colombia. Por otro lado, es probable que en esta área el diagnóstico de hospitalización y el acto quirúrgico sean percibidos como el foco global del manejo en deterioro de la prevención de la tromboembolia venosa, a diferencia del área médica, donde las enfermedades concomitantes y la necesidad de tratamientos simultáneos son mucho más frecuentes. Además, puede haber una mayor percepción en el área quirúrgica de dificultad en la evaluación del riesgo de tromboembolia venosa o de la probabilidad de sangrado o caídas y de posibilidad de efectos iatrogénicos con la profilaxis farmacológica, aunque paradójicamente estos argumentos han sido más utilizados para explicar las tasas bajas de profilaxis en pacientes de tratamiento médico en el resto del mundo (15).

Es probable que en la muestra colombiana de este estudio, se les haya prescrito profilaxis a más pacientes sin riesgo del área médica cuando no se iban a beneficiar de ella, en comparación con el área quirúrgica. Este hallazgo ya ha sido descrito anteriormente $(16,17)$. Infortunadamente, este estudio, a diferencia de IMPROVE $(6,8)$, no cuantificó la tasa de profilaxis en pacientes sin suficiente riesgo según los criterios del American College of Chest Physicians. Es muy probable que las tasas razonables de profilaxis en pacientes de tratamiento médico con riesgo de tromboembolia venosa en Colombia y en otros países, se asocien con exceso de prescripción inapropiada en aquellos sin suficiente riesgo, como fue documentado para Colombia en IMPROVE (6) y en otro estudio publicado recientemente (18). Esto podría sugerir que los médicos internistas, a diferencia de los cirujanos, le asignan más valor a prevenir la tromboembolia venosa, así se incurra en la profilaxis de algunos pacientes que no se beneficien de ella.

Por otro lado, es posible que algunas estrategias "genéricas" para mejorar la profilaxis de la tromboembolia venosa en pacientes hospitalizados puedan aumentar simultáneamente la profilaxis en pacientes que se beneficien y en aquellos que no se beneficien, aunque es muy probable que tal estrategia no aumente el riesgo de efectos adversos en los pacientes que reciben la profilaxis aun sin tener indicación por su bajo nivel de riesgo de tromboembolia venosa. En el estudio IMPROVE no se evidenció mayor sangrado en los pacientes colombianos y se sabe que el perfil de seguridad de las estrategias actuales de profilaxis es alto, pero podría ser fuente injustificada de mayores costos.

Es de anotar que las tasas apropiadas de profilaxis de la tromboembolia venosa, inclusive la de pacientes de tratamiento médico agudamente enfermos en Colombia, no alcanzan niveles lo suficientemente satisfactorios. El reto sigue siendo diseñar estrategias para mejorar estas tasas, pero reduciendo simultáneamente la profilaxis en los que claramente no se benefician de ella (6). Para esto, se necesitan estrategias educativas que promuevan la utilización sistemática de herramientas adecuadamente validadas para la estratificación del riesgo -las cuales siguen haciendo falta $(19,20)$-, involucrar el personal de salud diferente a los médicos (21) y establecer nuevas formas de promover la utilización de estas herramientas, probablemente basadas en avisos de alarma cuando se utilizan historias clínicas electrónicas (22-24).

En conclusión, el estudio ENDORSE demuestra que en los centros colombianos participantes se hizo más profilaxis apropiada contra la tromboembolia venosa en pacientes de tratamiento médico en riesgo, que en la gran mayoría de los países participantes. Este no es el caso en los pacientes colombianos de tratamiento quirúrgico, en quienes, a pesar de tener mayor riesgo que el promedio de los de tratamiento quirúrgico de los 32 países participantes, la tasa de profilaxis apropiada no estuvo entre las más altas. Se 
requieren todavía más esfuerzos en Colombia para mejorar la tasa de profilaxis de los pacientes hospitalizados de tratamiento quirúrgico o médico, pero simultáneamente, disminuyendo la profilaxis indiscriminada.

\section{Agradecimientos}

A laboratorios Sanofi-Aventis, por la financiación irrestricta de este proyecto. A todos los coordinadores y recolectores de información en los centros participantes, sin los cuales este estudio no hubiese sido posible.

\section{Conflictos de intereses}

Ninguno de los autores reporta conflictos de interés relacionados con este estudio.

\section{Financiación}

Laboratorios Sanofi-Aventis financió todos los aspectos relacionados con entrenamiento de los coordinadores y la recolección de la información en cada centro colombiano.

\section{Referencias}

1. Dennis R, Rojas MX, Molina A. Curso clínico y supervivencia en embolia pulmonar: resultados del registro multicéntrico colombiano (EMEPCO). Acta Méd Colomb. 2008;33:111-6.

2. Nieto JA, Monreal M, RIETE Investigators. Recurrent venous thromboembolism in men and women. $\mathrm{N}$ Engl $\mathrm{J}$ Med. 2004;351:2015-8.

3. Abuabara J, Acero R, Archila PE, Alí A, Bermúdez $\mathbf{M}$, Bohórquez R, et al. II Consenso colombiano de tromboembolismo venoso. Acta Méd Colomb. 2005;30:241-6.

4. Geertz WH, Bergqvitz D, Pineo GF. Prevention of venous thromboembolism. En: The 8th ACCP Conference on Antithrombotic and Thrombolitic Therapy. Chest. 2008;133:381S-453S.

5. Nicolaides AN, Fareed J, Kakkar AK. Prevention and treatment of venous thromboembolism. International consensus statement. Int Angiol. 2006;25:101-61.

6. Dennis R, Acevedo JR, Restrepo H. ¿Es apropiada la profilaxis actual del tromboembolismo venoso en pacientes médicos? Evaluación de desenlaces colombianos en el Registro Internacional sobre Prevención Médica del TEV (IMPROVE). Acta Méd Colomb. 2009;34:11-6.

7. Dennis R, Niño de A MN, Salazar MS. Encuesta nacional sobre tromboembolismo venoso en población hospitalaria. Acta Méd Colomb. 1996;21:55-63.

8. Tapson VF, Decousus H, Pini M. VTE prophylaxis in acutely ill hospitalized medical patients: Findings from the international medical prevention registry on VTE. Chest. 2007;132:936-45.
9. Cohen AT, Tapson VF, Bergmann JF. Venous thromboembolism risk and prophylaxis in the acute hospital care setting (ENDORSE study). Lancet. 2008:371:387-94.

10. Samama MM, Cohen AT, Darmon JY. A comparison of enoxaparin with placebo for the prevention of VTE in acutely ill medical patients. N Eng J Med. 1999;341:793-800.

11. Leizorovicz A, Cohen AT, Turpie AG. A randomized placebo controlled trial of dalteparin for the prevention of VTE in acutely ill medical patients. Circulation. 2004;110:874-9.

12. Cohen AT, Davidson BL, Gallus A. Efficacy and safety of fondaparinux for the prevention of VTE in older acute medical patients. BMJ. 2006;332:325-9.

13. Geertz WH, Pineo GF, Heit JA. Prevention of venous thromboembolism: The Seventh ACCP Conference on Antithrombotic and Thrombolytic Therapy. Chest. 2004;126:338S-400S.

14. Kahn SR, Panju A, Geerts W. Multicenter evaluation of the use of VTE profilaxis in acutely ill medical patients in Canada. Thromb Res. 2007;119:145-55.

15. Menajobsky LB, Spandorfer J. Benefits of more aggressive VTE prophylaxis in hospitalized medical patients. Cleve Clin J Med. 2004;71:947-8.

16. Scaglione L, Piobbici M, Pagano E. Implementing guidelines for VTE profilaxis in a large Italian teaching hospital: Lights and shadows. Haematologica. 2005;90:67884.

17. Chopard P, Dorffler-Melly J, Hess U. Venous thromboembolism prophylaxis in acutely ill medical patients: Definite need for improvement. J Intern Med. 2005;257:352-7.

18. Hernández J, Dennis R, Londoño D. Evaluación de la concordancia entre estrategias de profilaxis de TEV en paciente médico en un hospital universitario, Bogotá D.C., 2007-2008. Acta Méd Colomb. 2009;34:4-10.

19. Millar JA. Selection of medical patients for prophylaxis of venous thromboembolism based on analysis of the benefithazard ratio. Intern Med J. 2009;39:606-12.

20. Douketis JD. Prevention of venous thromboembolism in hospitalized medical patients: Addressing some practical questions. Curr Opin Pulm Med. 2008;14:381-8.

21. Labarere J, Bosson JL, Sevestre MA. Intervention targeted at nurses to improve venous thromboprophylaxis. Int J Qual Health Care. 2007;19: 301-8.

22. Kucher N, Koo S, Quiroz N. Electronic alerts to prevent VTE among hospitalized patients. N Eng J Med. 2005;352:96977.

23. Pham DQ, Pham AQ, Ullah E. Evaluating the appropriateness of thromboprophylaxis in an acute care setting using a computerised reminder, through order-entry system. Int J Clin Pract. 2008;62:134-7.

24. Piazza G, Goldhaber SZ. Improving clinical effectiveness in thromboprophylaxis for hospitalized medical patients. Am J Med. 2009;122:230-2. 